\title{
LIMITATION ASSESSMENT AND WORKFLOW REFINEMENT OF THE MANGROVE VEGETATION INDEX (MVI)-BASED MAPPING METHODOLOGY USING SENTINEL-2 IMAGERY
}

\author{
M. P. Neri ${ }^{1}$, A. B. Baloloy ${ }^{1}$, A. C. Blanco ${ }^{1,2}$ \\ ${ }^{1}$ Department of Geodetic Engineering, University of the Philippines, Diliman, 1101, Philippines - (mpneri1, abbaloloy, \\ acblanco)@up.edu.ph \\ ${ }^{2}$ Training Center for Applied Geodesy and Photogrammetry, University of the Philippines, Diliman, 1101, Philippines
}

KEY WORDS: mangroves, MVI, Sentinel-2, vegetation mapping, threshold

\begin{abstract}
:
The Mangrove Vegetation Index (MVI) was developed to map mangroves extent from remotely-sensed imageries accurately and quickly. MVI measures the probability of a pixel to be a 'mangrove' by extracting the greenness and moisture information from the green, NIR, and SWIR bands. The range of MVI values may vary depending on factors such as land cover classes, climatic conditions, or tidal conditions. Mapping the scope of mangrove sites involves setting a maximum and minimum MVI threshold to separate them from other land cover classes and vegetation. Although the MVI has a high index accuracy, its mapping performance is limited by some biophysical and environmental factors. Misclassification occurs in aquacultural areas, irrigated croplands, and sites with palm trees where mangrove and surrounding vegetation pixels have highly similar spectral signatures. There are scenes with complex environments, such as in aquaculture areas and along a network of rivers and streams, where an optimal threshold varies across the site, and setting a single MVI threshold may not yield excellent results. An automated threshold setting using the Otsu method was explored; however, the results were inaccurate due to a low intensity contrast between mangroves and other vegetation in the MVI raster layer. This study also looked into possible adjustments to improve the manual threshold setting workflow for a successful mapping of mangrove extent using MVI on Sentinel-2 imagery.
\end{abstract}

\section{INTRODUCTION}

Mangroves are an important component of various tropical ecosystems, providing breeding grounds for aquaculture, acting as coastal frontiers to stabilize shorelines, and acts as a carbon sink among others (Donato, 2011; Veettil 2019). However, despite their role and importance in nature being realized, mangrove ecosystems are one of the world's most threatened biomes, and losses are expected to continue as human populations increase (Long et al., 2014). Continuous refinement of mangrove mapping and monitoring methods is therefore crucial for the management of these important resources.

Baloloy et al. (2020) developed and proposed the Mangrove Vegetation Index (MVI), a new simplified index for fast and accurate mapping of mangrove extent from remotely-sensed images. The Mangrove Vegetation Index (MVI) equation is in the form:

$$
M V I=\frac{N I R-\text { Green }}{\text { SWIR } 1-\text { Green }}
$$

where NIR, Green, and SWIR1 are the band 8, band 3, and band 11 reflectance values of Sentinel-2 imagery. The | NIR - Green | enhances the differences of vegetation greenness between mangrove pixels and other vegetation, while | SWIR1 - Green | captures the distinct moisture of mangrove pixels apart from nonmangrove pixels. From the study sites, the highest MVI values recorded were those of mangrove pixels within the range of 4.5 to 16.5 followed by non-mangrove vegetation with a maximum threshold of 3.6. Other land classes such as clouds, water, builtup and soil has much lower MVI values. Hence, to separate

Corresponding author mangrove pixels from the other classes, an optimal minimum MVI threshold should be selected. MVI was found to successfully separate mangroves from other land cover with an index accuracy of $92 \%$.

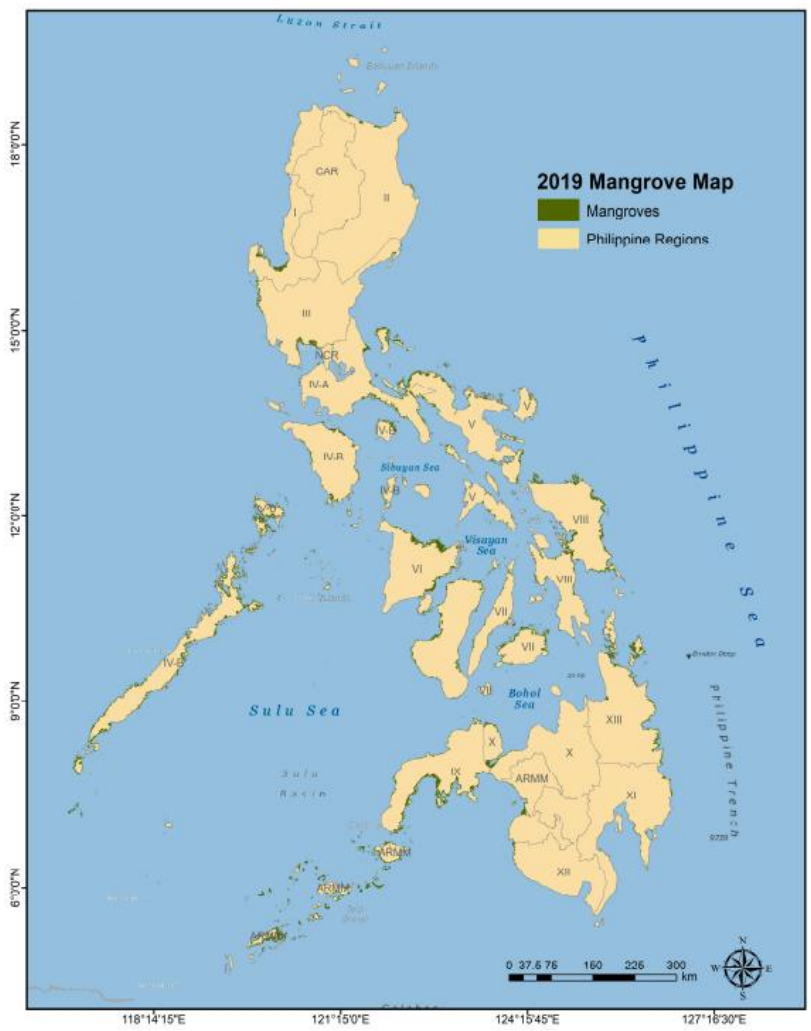

Figure 1. MVI-based 2019 Mangrove Extent Map of the Philippines (Baloloy et al., 2020). 
To facilitate faster mangrove extent mapping using the MVI, an Interactive Data Language (IDL)-based 'MVI Mapper' and an online MVI Mapper implemented in Google Earth Engine (GEE) were developed. These tools can be used for offline semiautomated processing and online fully-automated processing, respectively. The online MVI Mapper was used to generate the 2019 Mangrove Extent Map of the Philippines (Figure 1). The total mangrove cover in the Philippines in the 2019 map was computed to be 227,808 has.

This study aims to evaluate the limitations of the MVI in mangrove mapping, to conduct an assessment of the MVI-based 2019 Philippine Mangrove Extent Map, and to explore methods to improve threshold determination for a successful mapping of mangrove extent using MVI on Sentinel-2 imagery.

\section{DATA AND METHODS}

\subsection{Mapping the 2019 Philippine Mangrove Extent Map}

Baloloy et al. (2020) developed the online MVI Mapper, a fully automated online processing implemented on GEE for mapping of mangrove extent (Figure 2). In the MVI Mapper, the Philippines was divided into square regions or tiles using the Military Grid Reference System (MGRS). The mapper requires the following user inputs: MGRS tile code, start and end date of the desired Sentinel-2 imagery, the minimum and maximum MVI thresholds, and the output layers to display. The user can choose from any of the following products: RGB and false color composite (FCC) images, MVI vegetation raster, and MVI mangrove raster.

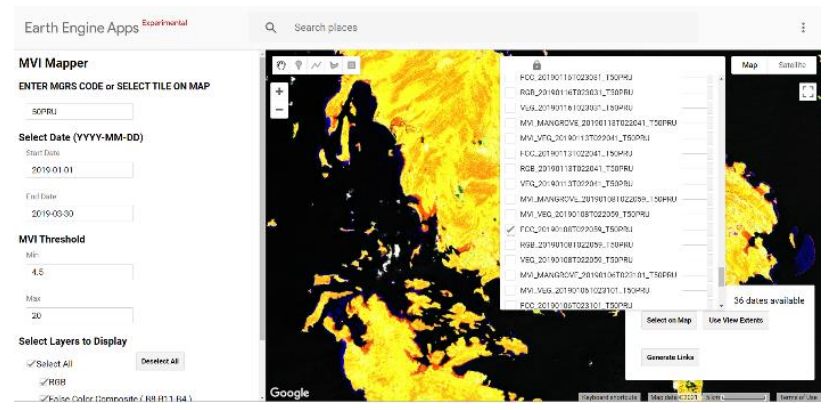

Figure 2. Google Earth Engine-based MVI Mapper interface.

The online MVI Mapper was used to generate the MVI-based 2019 Philippine Mangrove Extent Map. Atmospherically corrected Sentinel-2 images were used to generate the MVI raster layer per tile based on the identified optimal minimum threshold and a fixed maximum threshold of 20. Cleaning of noise pixels was done by overlaying the raster to the false color Sentinel-2 image with RGB B11-B8-B4. Out of 107 tiles covering the country, 87 were generated with MVI layers to produce the mangrove extent map.

\subsection{Map Limitation Assessment}

A new GEE script was developed to hasten the 2019 map product assessment. A polygon generated by the user enclosing the area of interest is first imported to the script. User inputs to be filled out are the date of the Sentinel image to be used for assessment, and the lower and upper MVI thresholds. There are 6 layers produced after running the script: the Sentinel-2 FCC, the MVI vegetation raster, the MVI mangrove raster based on the userinput threshold, the MVI mask raster based on the user-input threshold, the 2019 map product with filled mangrove pixels, and the 2019 map product with outlined mangrove pixels (Figure 3).
The area of mangrove extent based on the user-input threshold is automatically computed and displayed in the Console tab.

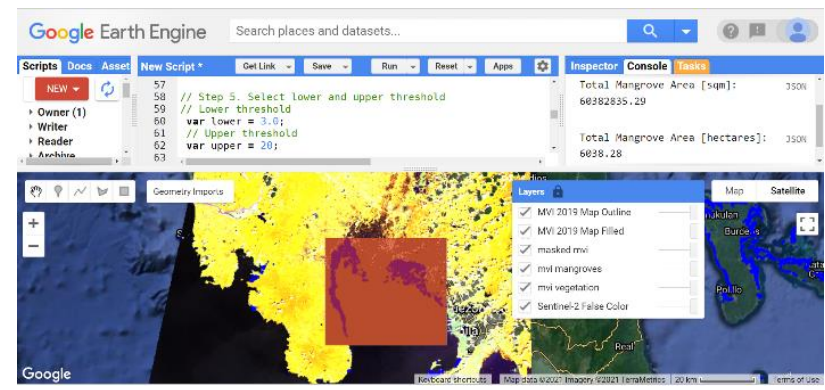

Figure 3. Products generated after running the GEE script to assess the MVI-based 2019 Mangrove Extent Map of the Philippines.

For assessment, these layers were viewed on top of each other. Google Earth satellite imagery and the FCC layer were used to verify mangrove pixels and sites. In identifying missed mangrove sites and misclassified mangrove pixels in the 2019 map, the MVI 2019 raster was viewed alternately over FCC and Google Earth imagery. Mangrove pixels appear in a bright orange color in FCC and has a texture distinct from other vegetation in Google Earth imagery. To determine the optimal minimum MVI thresholds per site, various threshold values were used to generate the MVI mask raster. The mask raster was inspected over FCC and Google Earth imagery in turns. The optimal threshold is selected based on which mapped out most of the mangrove pixels with the least number of misclassified pixels or noise.

\subsection{Workflow Refinement}

An automated image thresholding for MVI-based mangrove mapping using the Otsu method was explored. Otsu's method of thresholding directly operates on the gray level histogram to compute an optimized threshold value that separates objects from the background in an image (Bangare et al., 2015). This method of thresholding and image segmentation is primarily dependent on data intensity (Goh et al., 2017). For this MVI-based thresholding, the mangroves are the object, while the surrounding non-mangrove vegetation are the background pixels.

The GEE script for map limitation assessment was modified to accommodate the Otsu thresholding (Figure 4). The user is

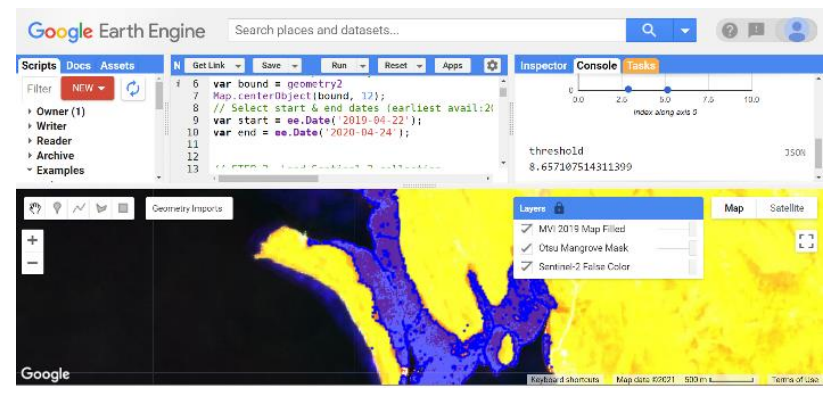

Figure 4. Products generated after running the script for the automatic identification of MVI threshold using Otsu method in GEE.

required to create two (2) geometries, one enclosing the region of interest, and another to enclose a subset with mangrove and other vegetation. The script generates an MVI raster with masked nonvegetation pixels for analysis. Three (3) layers are produced after running the script: the FCC, the MVI mask raster based on the 
automatically identified Otsu threshold, and the 2019 map product with filled mangrove pixels. The following statistics and charts are also displayed in the Console tab: the histogram of MVI values within the subset, the mean and variance of the MVI values within the subset, the Otsu threshold of the scene, and the background and object ratio.

FCC visualization and Google Earth satellite imagery were used as guides to locate sites with mangroves and other vegetation. The threshold identified using the Otsu method was compared against the assigned threshold used in the 2019 map.

\section{RESULTS AND DISCUSSION}

\subsection{Missed Mangroves}

The online MVI Mapper uses the Military Grid Reference System (MGRS) tiles to organize mapping of mangrove extents in the entire Philippines. It was utilized in generating the Philippine mangrove extent map of 2019. In examining this product, it was found that there are three (3) tiles without accounted mangroves. These are the tiles with codes 51NYJ, $51 \mathrm{NYH}$, and $51 \mathrm{NYG}$, all located in the southern region of the Philippines (Figure 5).

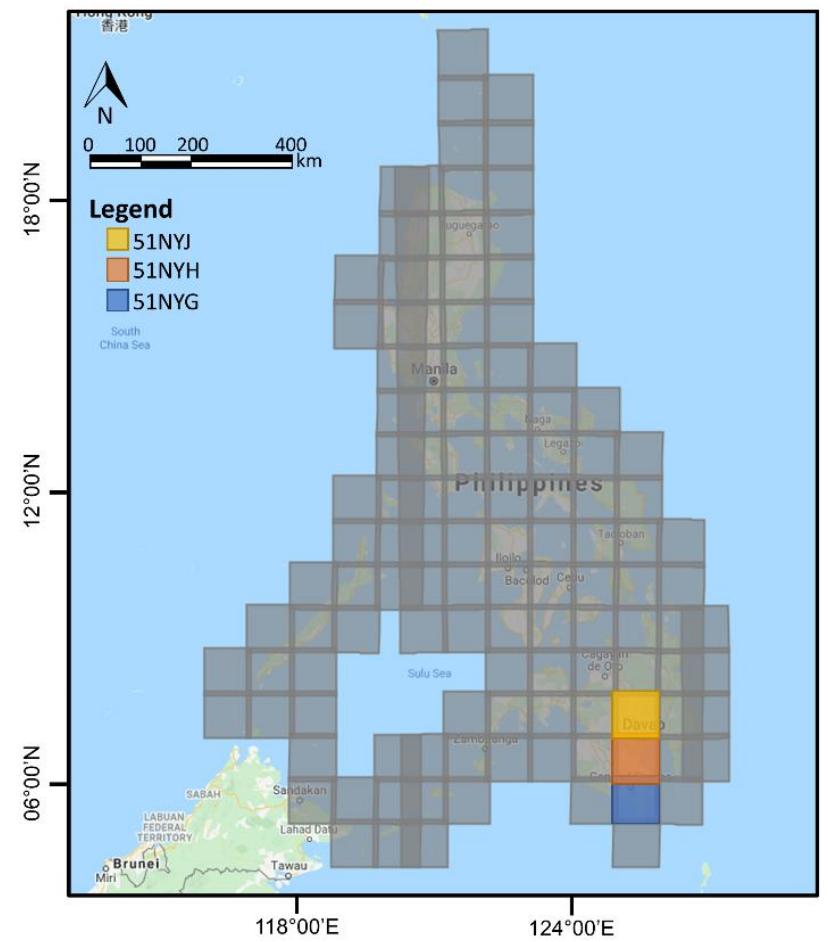

Figure 5. Map of Military Grid Reference System (MGRS) tiles in the online Mangrove Vegetation Index (MVI) Mapper with unaccounted mangroves in the 2019 Mangrove Extent Map of the Philippines produced using MVI.

Possible missed mangroves in the said tiles were searched using FCC with RGB B11-B8-B4 visualization of atmospherically corrected Sentinel-2 images. With FCC composite in the online MVI Mapper, mangroves are displayed in a distinct bright orange or red hue. Sites identified to be probable mangroves were verified using Google Earth satellite imagery.

In mapping the 2019 mangrove extent of the Philippines using MVI, a vegetation mask from Sentinel quality scene classification band was applied. Since most of the areas in tiles $51 \mathrm{NYJ}$ and $51 \mathrm{NYH}$ are located well in the middle of the large
Mindanao Island, mangroves in small lakes and along small streams within the said tiles were masked out. In tile 51NYJ, a distinct site with unaccounted mangroves is along Libuganon River in Davao Del Norte (Figure 6). Some sites with missed mangroves in tile $51 \mathrm{NYH}$ are those along the Davao Gulf shoreline (Figure 7).
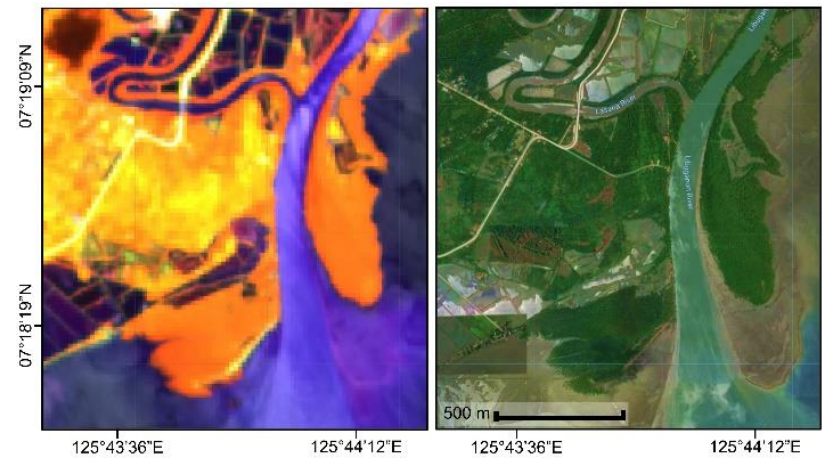

Figure 6. Mangroves along Libuganon River in tile 51NYJ that are unaccounted for in the MVI-based 2019 Mangrove Extent Map of the Philippines.
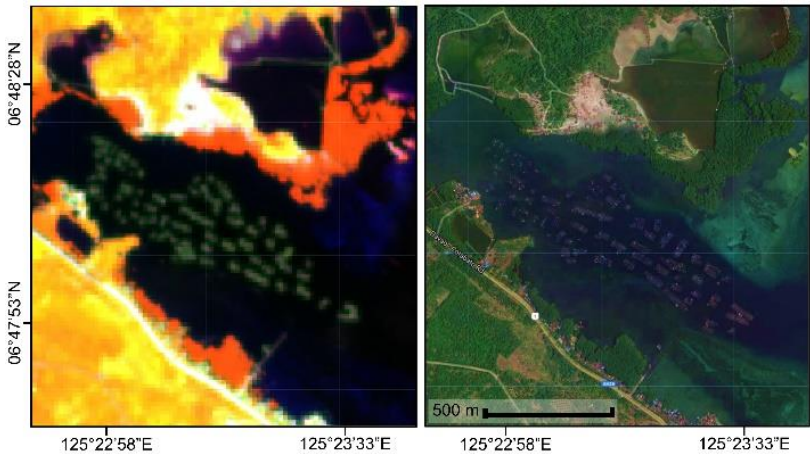

Figure 7. Mangroves along the Davao Gulf shoreline in Tile $51 \mathrm{NYH}$ that are unaccounted for in the MVI-based 2019 Mangrove Extent Map of the Philippines.

Compared against the other two, tile 51NYG has the most number and largest area of unaccounted mangrove sites. Mangroves are missed along the shorelines of bays and gulfs such as Sarangani Bay (Figure 8).

Upon a more thorough inspection of the tiles, the following minimum MVI thresholds were found to suit the tiles most optimally (Table 1). These thresholds were selected on the basis of being able to identify the largest possible mangrove area with the least noise or misclassified pixels. To verify the identified
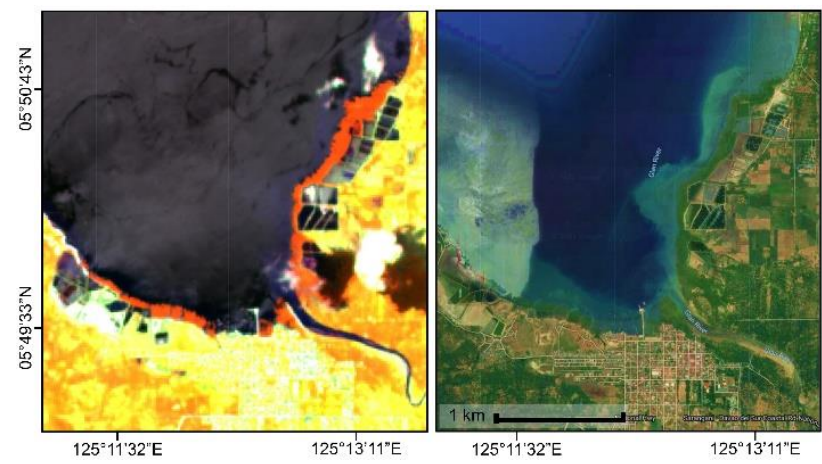

Figure 8. Mangroves along Sarangani Bay in tile 51NYG that are unaccounted for in the 2019 Mangrove Extent Map of the Philippines. 
mangrove areas, the MVI shapefiles were overlaid on top of the FCC and Google Earth satellite imagery.

\begin{tabular}{ccccc}
\hline $\begin{array}{c}\text { MGRS } \\
\text { Code }\end{array}$ & Year & Month & Day & $\begin{array}{c}\text { MVI } \\
\text { Threshold }\end{array}$ \\
\hline 51 NYJ & 2019 & 5 & 22 & 3.8 \\
$51 \mathrm{NYH}$ & 2019 & 6 & 6 & 3.7 \\
$51 \mathrm{NYG}$ & 2019 & 6 & 6 & 4.2 \\
\hline
\end{tabular}

Table 1. Suggested minimum threshold for the missed tiles in the 2019 Mangrove Extent Map of the Philippines.

Aside from those within unaccounted tiles, there are also missed mangroves in other tiles. Two of these mangrove sites are those surrounding Lanao Lake and along Mindanao River. There are also some mangrove sites missed along the shorelines such as mangroves along Looc Bay (Figure 9).
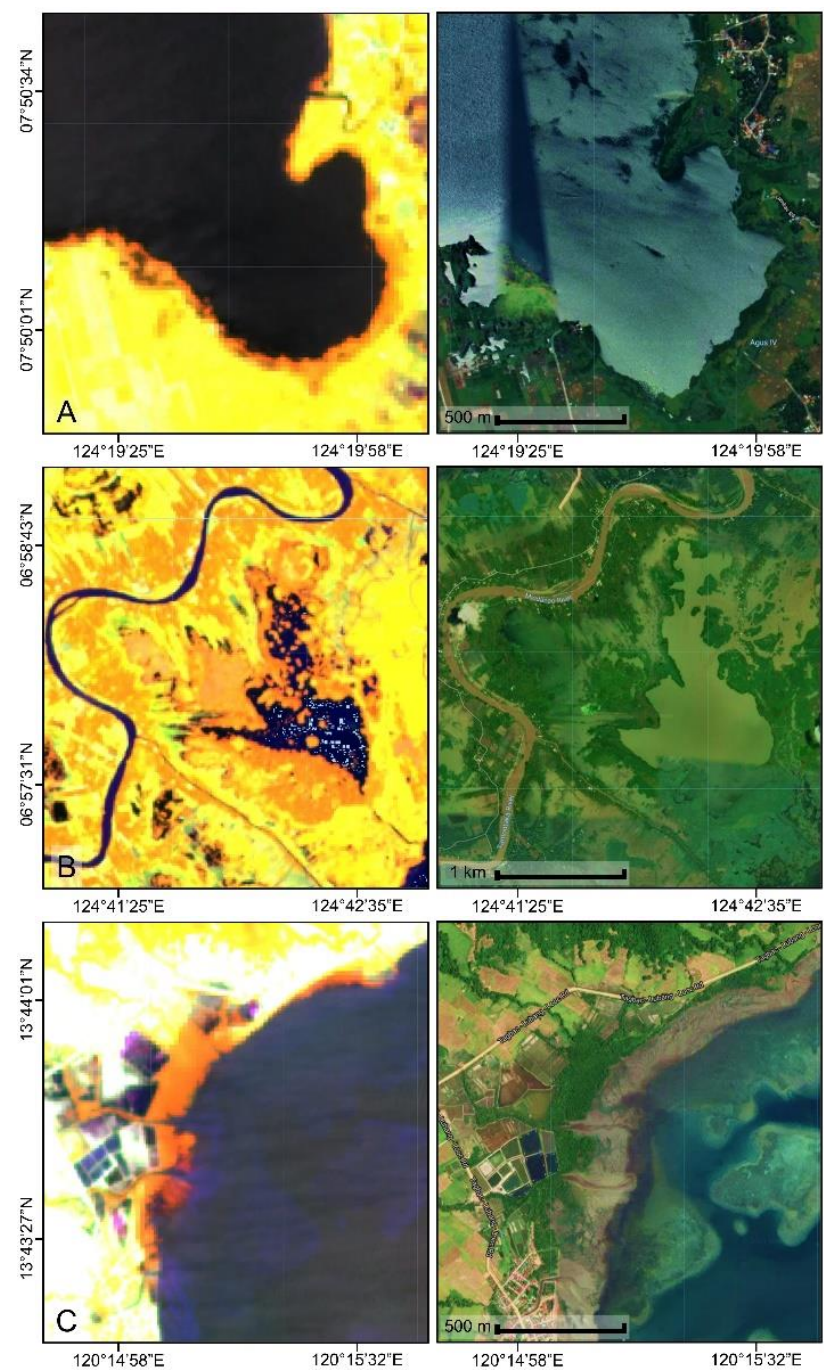

Figure 9. Missed mangroves in the 2019 MVI Mangrove Extent Map of the Philippines (A) surrounding Lanao Lake, (B) along Mindanao River, and (C) along Looc Bay.

\subsection{Sites Misclassified as Mangroves}

The 2019 MVI Mangrove Extent Layer was also scrutinized for sites and objects that are misclassified as mangroves. Portions of fishponds, agricultural areas with irrigated crops, and palm trees were found incorrectly recognized as mangroves (Figure 10).
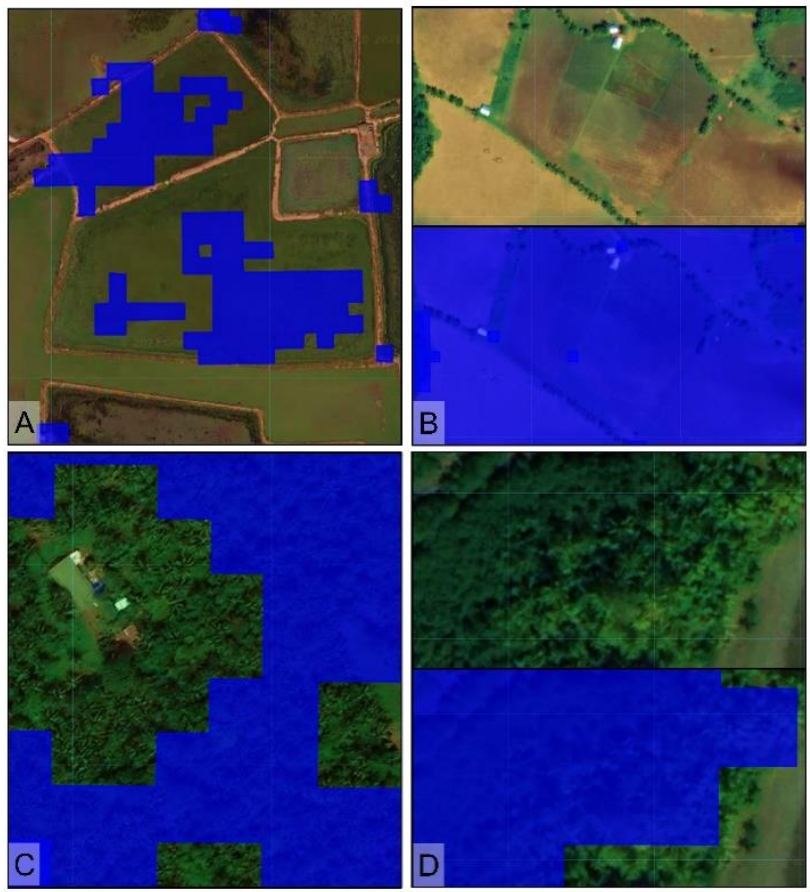

Figure 10. Sites misclassified as mangroves: (A) aquaculture ponds, (B) croplands, (C) coconut trees, and (D) Nypa fruticans. The blue pixels are areas classified as mangroves in the MVIbased 2019 Mangrove Extent Map of the Philippines.

3.2.1 Misclassification in Fishponds: Mangroves grow on fishponds and dikes as a result of silvofisheries, a system of combining the fishpond with mangrove trees. There are shallow fishponds misclassified as mangroves in the 2019 map. Pixels which are a mixture of natural non-mangrove vegetation and water become misclassified as mangrove pixels due to a high degree of their spectral similarity that makes them difficult to be identified as separate classes (Hu et al., 2020). Shrubs on dikes alongside mangroves are also commonly mistakenly identified as mangroves. The misclassification in these areas are expected to be reduced as mangroves densify (Chen et al., 2017).

3.2.2 Misclassification in Croplands: Irrigated crops misclassified as mangroves are rarely found in the 2019 map, and are isolated cases of small areas. Misclassified areas were observed to be near the vicinity of large clusters of mangroves. The spectral similarity between some mangrove forests and nearby croplands in single-date satellite imageries makes accurate extraction of mangrove pixels complicated (Hu et al., 2018).

3.2.3 Misclassified Palm Trees: Extensive areas of coconut tree clusters misclassified as mangroves were identified mostly in the Samar-Leyte regions.

A factor that seemingly contributed significantly to the misclassification of coconut trees in the 2019 map is the use of FCC for verification of mangrove areas. Looking closely at the true color composite in Figure 11, there is a clear distinction between the textures of the mangrove areas and the areas dominated by coconut trees - mangroves appear with a finer texture while coconut trees appear rougher. On the other hand, using the FCC visualization, there is low contrast between the hues of the mangrove areas and the coconut trees. Scrutinizing the hues more carefully, mangrove areas appear in the darkest orange while the coconut tree areas appear in yellow gold color. 

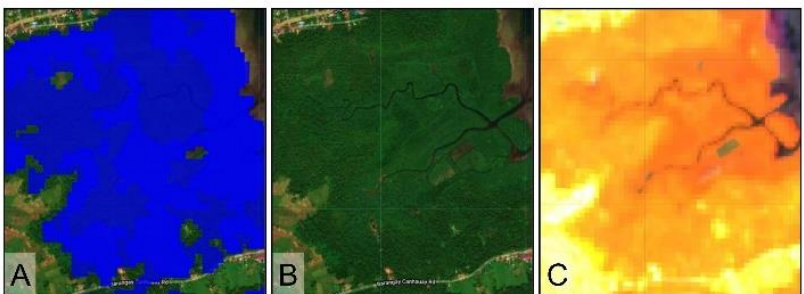

Figure 11. Misclassified coconut trees as mangroves: (A) The blue pixels are those that are classified as mangroves, (B) true color composite, and (C) FCC, where mangroves are represented by the darker orange color.

In Figure 12A, the mangroves and coconut trees can be identified distinctively from each other solely based on their textures. Utilizing a higher value for minimum MVI threshold identifies less coconut trees as mangroves; however, the results still does not show a clear distinction on the mangrove bounds.

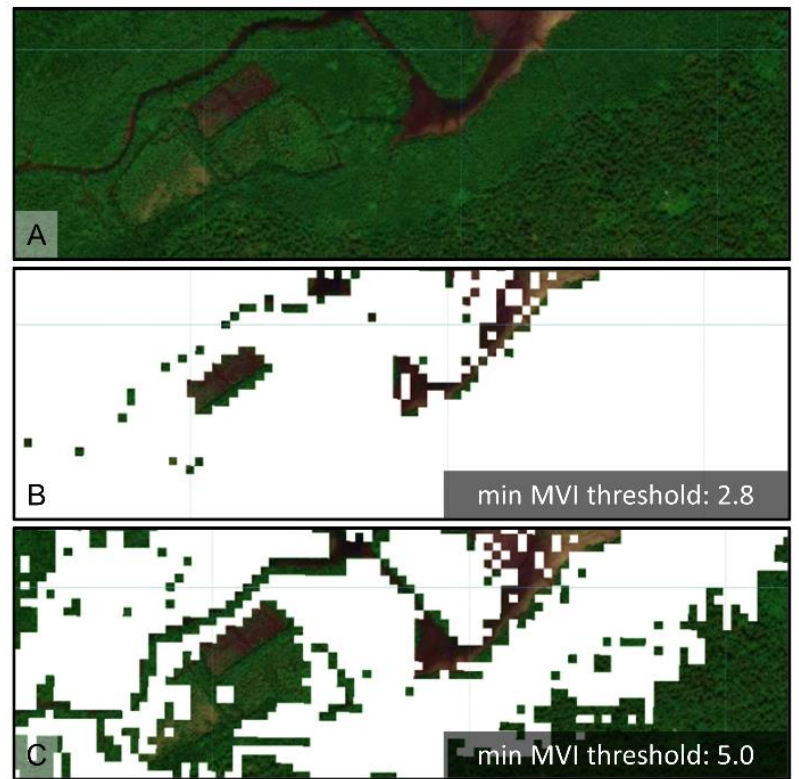

Figure 12. Adjusted minimum threshold to reduce misclassified coconut trees on (A) true color composite: the white pixels are

those identified to be mangroves using minimum MVI thresholds of (B) 2.8 as used for the MVI-based 2019 Mangrove Extent Map of the Philippines and (C) 5.0.

Another type of palm trees misclassified as mangroves in the 2019 map are the Nypa fruticans. Nypa fruticans (family Arecaceae), although considered mangroves, do not possess primary characteristics of mangroves as they don't exploit littoral environments, and they cannot endure inundation with undiluted sea-water for long periods (Joshi et al., 2006). The nypas appear in thin strips alongside mangroves if not mixed within clusters of mangroves. Unlike coconut trees, the MVI and FCC signature of nypas appear similar with surrounding mangroves and adjusting the minimum threshold does not result to less misclassified nypas. The difficulty in distinguishing mangroves form nypas are mainly driven by similar spectral properties and the natural mixing of these vegetation especially within fringe mangrove forests (Nwobi et al., 2020).

\subsection{Varying Thresholds}

3.3.1 Threshold Setting in Complex Environments: In assigning thresholds, the main consideration is to map out most of the mangrove pixels with the least misclassified pixels or noise. In setting the minimum MVI threshold, however, there are scenes with complex environments where setting a single MVI threshold may not yield excellent results.

Most sites found to have varying thresholds are in aquaculture areas. Figure 13 depicts how different thresholds affect the classification in this type of environment.
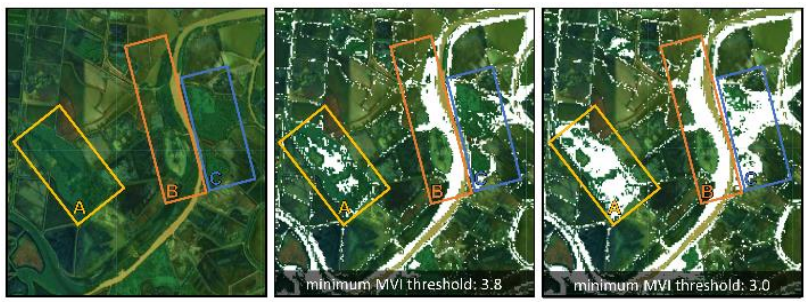

Figure 13. Varying thresholds in a complex environment for clusters of mangroves in separate regions A (yellow), B (orange), and $\mathrm{C}$ (blue). The white pixels are those identified to be mangroves for the case MVI $\geq 3.8$ (middle) and for the case $\mathrm{MVI} \geq 3.0$ (right).

In the 2019 map, the minimum MVI threshold set for this site in Aklan Province is 3.8. Using the said threshold, the mangroves on the dike in region B were optimally classified and its bounds were well delineated. Most mangroves in regions A and C, however, were missed. Setting a lower threshold yields more pixels classified as mangroves. Using a lower threshold of 3.0, more pixels are correctly classified as mangroves, but there are lots of noise introduced in the surrounding areas.

In another site in Zamboanga Sibugay, the MVI threshold used in the 2019 map is 3.1 (Figure 14). Mangroves in region B were well classified and delineated using this threshold, but there were missed mangroves in region A and misclassified pixels in region C. Setting a higher threshold of 5.0 resulted to less misclassified pixels in region $\mathrm{C}$, but more mangroves missed in region $\mathrm{B}$. On the other hand, using a lower threshold of 2.0 yields more pixels correctly classified as mangroves in region $\mathrm{A}$ and more noise introduced in the surrounding areas.

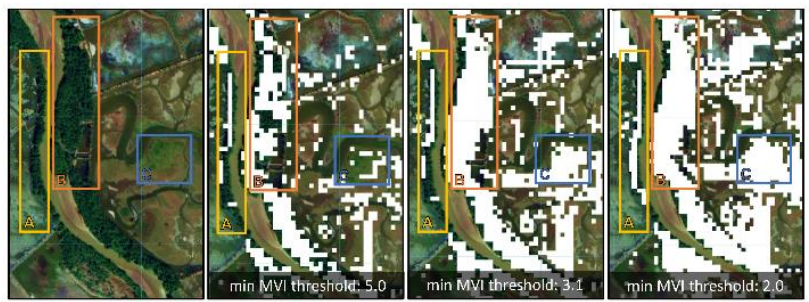

Figure 14. Varying thresholds in a complex environment for mangroves in regions A (yellow) and B (orange), and moss in region $\mathrm{C}$ (blue). The white pixels are those identified to be mangroves for the case MVI $\geq 5.0$ (middle left), MVI $\geq 3.1$ (middle right), and $\mathrm{MVI} \geq 2.0$ (right).

3.3.2 Site-Specific Thresholds in a Single Tile: In mapping the mangroves in the 2019 MVI Mangrove Extent Map of the Philippines, a single MVI threshold is assigned per MGRS tile. One tile covers an area of 10,000 sq. $\mathrm{km}$. These thresholds, however, do not always work when working on smaller regions.

In Figure 15 are two (2) sites in tile 51PTS with an assigned threshold of 3.0 in the 2019 map. Both of these are dominated by aquacultural areas. Exploring these, it was found that a threshold of 3.2 works better for Site A and the original threshold of 3.0 for Site B. 


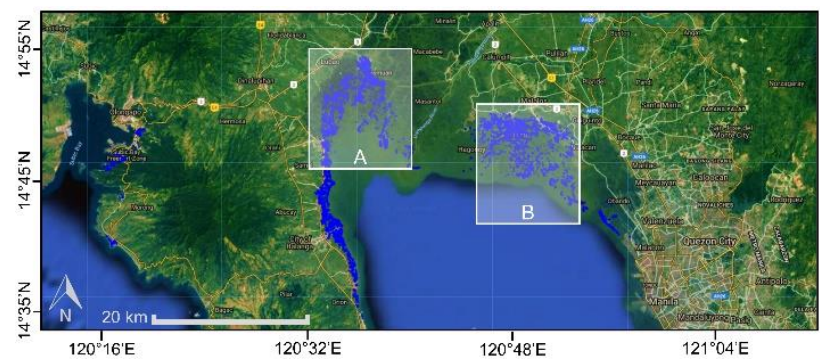

Figure 15. Sites with varying thresholds in tile 51PTS: (A) Masantol, Pampanga to Orion, Bataan, and (B) Hagonoy and Paombong, Bulacan. The blue pixels are classified as mangroves in the MVI-based 2019 Mangrove Extent Map of the

Philippines. The computed areas of mangrove cover in these sites using the original and optimal site-specific thresholds are summarized in Table 2.

Figure 16 shows three (3) sites in tile 51NVJ with a threshold of 3.1 in the 2019 map. Scrutinizing these regions closely showed that mangroves were better mapped using threshold values of 3.1 for Site A, 3.4 for Site B, and 3.3 for Site C. The original assigned threshold for Sites B and C misclassified large areas with coconut trees as mangroves. A higher threshold value reduces the misclassified pixels in these regions significantly.

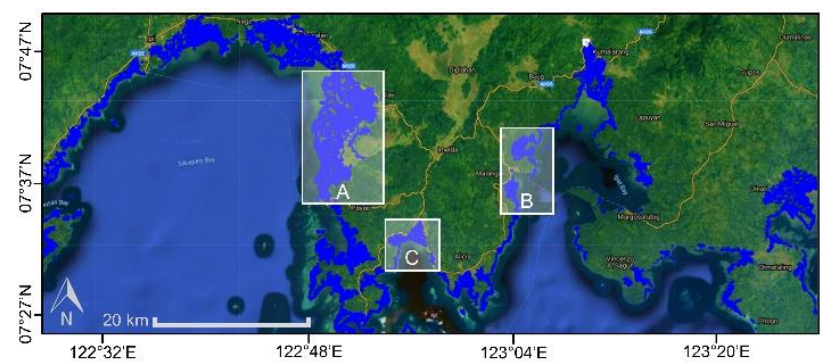

Figure 16. Sites with varying thresholds in tile 51NVJ: (A) near Sibuguey Bay, (B) near Igat Bay, and (C) near Tantanang Bay. The blue pixels are classified as mangroves in the MVI-based 2019 Mangrove Extent Map of the Philippines. The computed areas of mangrove cover in these sites using the original and optimal site-specific thresholds are summarized in Table 2.

In Figure 17, the optimal thresholds in the two sites vary since they have different dominating features - coconut trees in Site A, and Nypa fruticans in Site B. The 2019 map threshold used is 2.8, however, a closer inspection suggested that a higher threshold of 5.0 yields better results in site A since lots of coconut trees are misclassified using the original threshold.

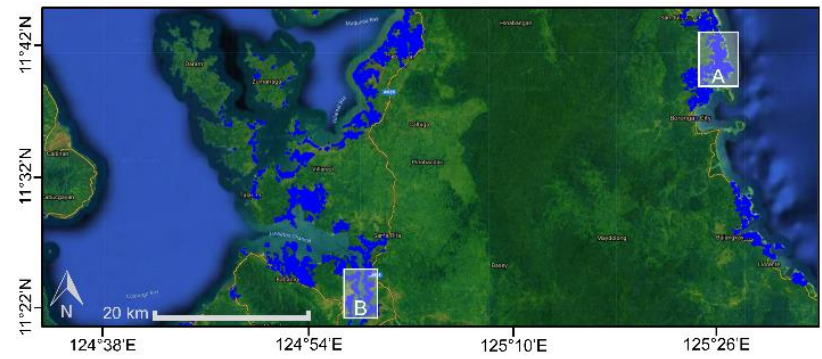

Figure 17. Sites with varying thresholds in tile 51PYN: in (A) Canhauay, Eastern Samar, and along (B) San Juanico Strait. The blue pixels are classified as mangroves in the MVI-based 2019 Mangrove Extent Map of the Philippines. The computed areas of mangrove cover in these sites using the original and optimal site-specific thresholds are summarized in Table 2.
Assigning new MVI thresholds meant the number of pixels classified as mangroves is altered, and so is the computed area. Table 2 summarizes the computed areas in these sites using their original assigned MVI thresholds in generating the 2019 map and the updated ones.

\begin{tabular}{ccccccc}
\hline Site & & Tile & $\begin{array}{c}\text { Original } \\
\text { Threshold }\end{array}$ & $\begin{array}{c}\text { Area } \\
\text { (has.) }\end{array}$ & $\begin{array}{c}\text { Optimal } \\
\text { Threshold }\end{array}$ & $\begin{array}{c}\text { Area } \\
\text { (has.) }\end{array}$ \\
\hline $\begin{array}{c}\text { Fig. } \\
15\end{array}$ & A & 51PTS & 3.0 & 1579.21 & 3.2 & 1430.53 \\
& B & & & 1199.96 & 3.0 & 1199.96 \\
\hline $\begin{array}{c}\text { Fig. } \\
16\end{array}$ & A & \multirow{2}{*}{51 NVJ } & \multirow{2}{*}{3.1} & 3297.96 & 3.1 & 3297.96 \\
& B & & & 807.05 & 3.4 & 658.20 \\
& C & & & 557.36 & 3.3 & 452.75 \\
\hline Fig. & A & \multirow{2}{*}{51 PYN } & \multirow{2}{*}{2.8} & 1571.83 & 5.0 & 233.62 \\
17 & B & & & 792.33 & 2.8 & 792.33 \\
\hline
\end{tabular}

Table 2. Comparison of computed areas of mangrove cover using the original and optimal site-specific thresholds in the sites with varying thresholds in Figures 15, 16, and 17.

\subsection{Workflow Refinement}

Manual thresholding is dependent on the judgment of the observer and assigning an optimal threshold can vary from person to person. In this study, an automated identification of an optimal minimum MVI threshold using the Otsu method within a site is explored.

The results generated using the automated Otsu thresholding were significantly higher than the assigned MVI thresholds from the 2019 map. Using the Otsu threshold, there was a clear underclassification of mangrove pixels when viewed on top of the FCC (Figure 18). Mangrove pixels in FCC appear bright orange, while non-mangrove pixels are yellow.

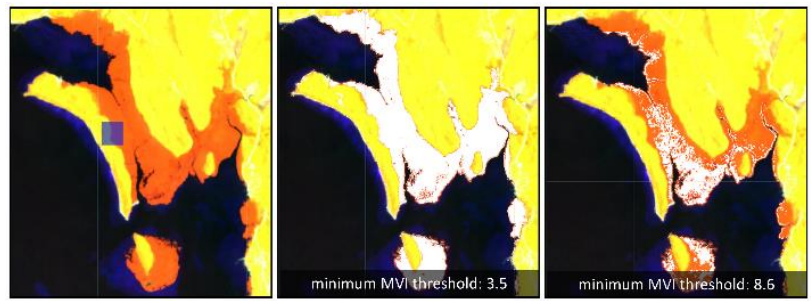

Figure 18. Comparison of thresholds from the MVI-based 2019 Mangrove Extent Map of the Philippines and the automated Otsu method: FCC and the subset for Otsu analysis in the blue geometry (left), for the case MVI $\geq 3.5$ from the 2019 map (middle) and for the case MVI $\geq 8.6$ as determined using the Otsu method (right). The white pixels are those identified to be mangroves based on the threshold.

The poor results from the automated Otsu thresholding may be accounted to the very low contrast between mangroves and surrounding vegetation when viewing the MVI layer. As shown in Figure 19, the mangrove area, depicted in bright orange in the FCC, is distinct from surrounding vegetation; however, the MVI layer shows little to no difference between the two. The effectiveness of the Otsu technique relies greatly on the contrast between intensities of the background and object (Bangare et al., 2015). The precision of image segmentation in this method cannot be obtained when the grey level of objects is close to that of the background (Yuan et al., 2016). To improve the automation of identifying the optimal threshold using the Otsu 
method, contrast enhancement of the MVI layer should be explored further.

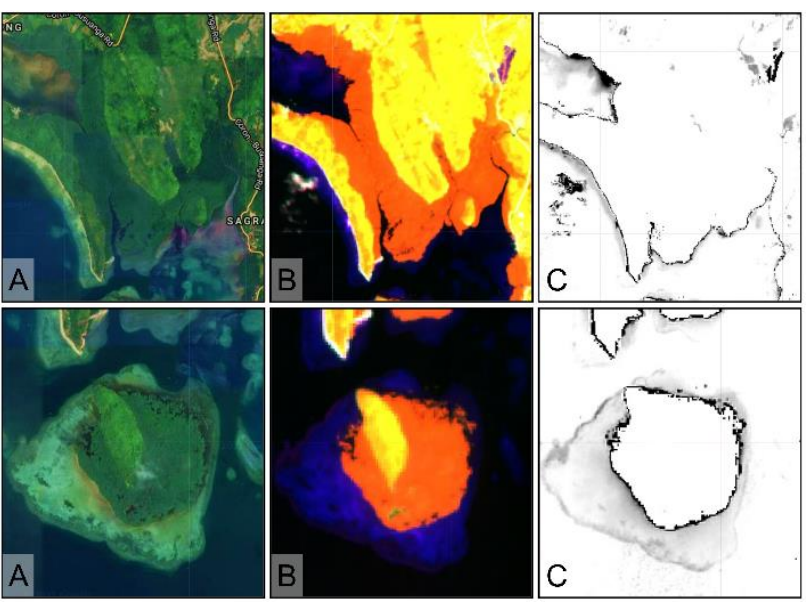

Figure 19. Contrast visualization of mangroves against other vegetation in (A) Google Earth satellite imagery, (B) FCC, and (C) MVI layer.

Since there are still improvements to be done in the automated determination of optimal MVI thresholds, possible adjustments to improve the manual threshold assignment were looked into. First, masking non-vegetation pixels, especially water pixels, was found to aid in minimizing noise pixels when generating the MVI raster. Second, misclassification in mapping coastal vegetation like mangrove forests may be reduced by ensuring that satellite data obtained is during low tide to eliminate effects of tidal inundation (Chen et al., 2017). Tidal inundation affects the spectral signature of very sparse mangroves in such a way that the spectrum of mangroves becomes similar to that of water when water level is high (Hu et al., 2018). Lastly, masking by slope can be applied since mangrove forests occur in wetlands with relatively flat topography (Chen et al., 2017).

\section{CONCLUSION}

This study assessed the MVI for mapping mangrove extent, as well as the MVI-based 2019 Mangrove Extent Map of the Philippines in the research work by Baloloy et al. (2020). In the 2019 map, most of the missed mangroves were masked out due to the application of a vegetation mask from Sentinel quality scene classification band. Misclassification was found to occur in sites such as fishponds, croplands near mangrove sites, and areas in or near coastal regions with palm trees due to a high degree of spectral similarity between mangroves and other vegetation in these sites. A crucial limitation of MVI mapping is threshold setting in complex mangrove environments such as fishponds and those nearby a network of rivers and streams. In these environments, optimal thresholds vary across the site, and a single MVI threshold may not yield excellent results. MVI thresholds are also site-specific, hence, an assigned optimal threshold for a large site do not always work for its subset regions. To hasten the MVI threshold selection in mangrove mapping, an automated thresholding using the Otsu method was explored. The results, however, were inaccurate due to a low gray-level contrast between mangroves and surrounding vegetation. A possible improvement for this automation is contrast enhancement of the MVI layer. To refine MVI-based mapping using manual thresholding, recommended adjustments are masking water pixels to minimize noise, utilizing satellite data captured during low-tide to eliminate tidal inundation effects and reduce misclassification, and to mask by slope.

\section{ACKNOWLEDGEMENTS}

This research work was done under the Comprehensive Assessment and Conservation of Blue Carbon Ecosystems and their Services in the Coral Triangle (BlueCARES) Project funded by the University of the Philippines Diliman and the Japan International Cooperation Agency (JICA).

\section{REFERENCES}

Baloloy, A.B., Blanco, A.C., Sta. Ana, R.R.C., Nadaoka, K. 2020. Development and application of a new mangrove vegetation index (MVI) for rapid and accurate mangrove mapping. ISPRS Journal of Photogrammetry and Remote Sensing. 166, 95-117. https://doi.org/10.1016/j.isprsjprs.2020.06.001

Bangare, S.L., Amruta, D., Bangare, P.S., Patil, S.T. 2015. Reviewing Otsu's Method For Image Thresholding. International Journal of Applied Engineering Research. 10 (9), 21777-21783. https://doi.org/ 10.37622/ijaer/10.9.2015.2177721783

Chen, B., Xiao, X., Lianghao, P. 2017. A mangrove forest map of China in 2015 Analysis of time series Landsat 78 and Sentinel$1 \mathrm{~A}$ imagery in Google Earth Engine cloud computing platform. ISPRS Journal of Photogrammetry and Remote Sensing. 131(1996),

https://doi.org/10.1016/j.isprsjprs.2017.07.011

104-120.

Donato, D.C., Kauffman, J.B., Murdiyarso, D., Kurnianto, S., Stidham, M., Kanninen, M. 2011. Mangroves among the most carbon-rich forests in the tropics. Nature Geoscience. 4, 293-297. https://doi.org/10.1038/ngeo1123

Goh, T.Y., Basah, S.N., Yazid, H., Safar, M.J.A., Saad, F.S.A. 2017. Performance analysis of image thresholding: Otsu technique. Measurement. $114 \quad$ (2018). 298-307. http://dx.doi.org/10.1016/j.measurement.2017.09.052

Hu, L., Xu, N., Liang, J., Li, Z., Chen, L., Zhao, F. 2018. Advancing the Mapping of Mangrove Forests at National-Scale Using Sentinel-1 and Sentinel-2 Time-Series Data with Google Earth Engine: A Case Study in China. 2020. Remote Sens. 12(19), 3120. https://doi.org/10.3390/rs 12193120

Joshi, L., Kanagaratnam, U., Adhuri, D. 2006. Nypa fruticans useful but forgotten in mangrove reforestation - useful but forgotten in mangrove reforestation programs?. Resilience, Rights and Resources: Two years of recovery In coastal zone Aceh. 1-4.

Veettil, B.K., Ward, R.D., Quang, N.X., Trang, N.T.T., Giang, T.H. 2019. Mangroves of Vietnam: Historical development, current state of research and future threats. Estuarine, Coastal $\begin{array}{llll}\text { and } \quad \text { Shelf } & \text { Science. 218, } 236 .\end{array}$ https://doi.org/10.1016/j.ecss.2018.12.021.

Nwobi, C., Williams, M., Mitchard, E.T.A. 2020. Rapid Mangrove Forest Loss and Nipa Palm (Nypa fruticans) Expansion in the Niger Delta, 2007-2017. Remote Sens. 12(14), 2344. https://doi.org/10.3390/rs12142344

Yuan, X., Martínez, J.-F., Eckert, M., López-Santidrián, L. 2016. An Improved Otsu Threshold Segmentation Method for Underwater Simultaneous Localization and Mapping-Based 
The International Archives of the Photogrammetry, Remote Sensing and Spatial Information Sciences, Volume XLVI-4/W6-2021

Philippine Geomatics Symposium 2021, 17-19 November 2021, virtual meeting
Navigation.
Sensors.
16
$1148-1179$

https://doi.org/10.3390/s16071148 